\title{
Fluxo de biomassa em capim-massai durante o estabelecimento e rebrotação com e sem adubação nitrogenada ${ }^{1}$
}

\author{
Marcos Neves Lopes², Magno José Duarte Cândido³, Roberto Cláudio Fernandes Franco Pompeu,, \\ Rodrigo Gregório da Silva ${ }^{5}$, José Wellington Batista Lopes ${ }^{6}$, Francisco Ronaldo Belém Fernandes ${ }^{6}$, \\ Claudivan Feitosa de Lacerda ${ }^{7}$,Francisco Marcus Lima Bezerra ${ }^{8}$
}

\section{RESUMO}

A produção de biomassa em uma pastagem pode ser incrementada com o uso de fertilizantes, principalmente os nitrogenados. Diante disso, objetivou-se, com este trabalho, avaliar o fluxo de biomassa do capim-massai, durante o estabelecimento e a rebrotação, em casa de vegetação, sob cinco doses de nitrogênio $(0 ; 150 ; 300 ; 450$ e 600 mg de N $\mathrm{dm}^{-3}$ de solo), em delineamento inteiramente casualizado, com medidas repetidas no tempo, e cinco repetições. A taxa de alongamento foliar foi incrementada pelas doses de nitrogênio $(\mathrm{N})$ e o crescimento de estabelecimento apresentou maior valor, em relação ao das rebrotações. A taxa de alongamento das hastes não foi influenciada pelas doses de N. O crescimento de estabelecimento apresentou maior valor para esta variável, em relação ao das rebrotações. A taxa de senescência foliar anterior não sofreu influência das doses de $\mathrm{N}$, porém, foi alterada entre os ciclos de crescimento. $\mathrm{O}$ número de folhas vivas por perfilho foi superior, para as maiores doses e no estabelecimento, o inverso ocorrendo para o filocrono. A dose aplicada de $600 \mathrm{mg} \mathrm{dm}^{-3}$ de $\mathrm{N}$ proporcionou um incremento de $448 \%$, na taxa de produção de forragem, e de $455 \%$, na taxa de acúmulo de forragem, em relação àquelas do manejo sem N. A adubação nitrogenada proporciona respostas positivas nas variáveis do fluxo de biomassa do capim-massai.

Palavras-chave: filocrono, morfogênese, nitrogênio, Panicum maximum.

\section{ABSTRACT}

\section{Response of biomass flow in Massai grass to nitrogen fertilization during the establishment and regrowth}

The use of fertilizers, especially nitrogen, can increase significantly the biomass production of a pasture. The objective of this study was to evaluate the biomass flow of Massai grass produced in greenhouse during the establishment and regrowth under five nitrogen fertilization levels. The experiment was arranged in a completely randomized split-plot design with five nitrogen levels $\left(0 ; 150 ; 300 ; 450\right.$ e $600 \mathrm{mg} \mathrm{de} \mathrm{N} \mathrm{dm}^{-3}$ of soil) and five replications (plots) .The leaf elongation rate was increased with the nitrogen fertilization levels and the establishment growth presented higher values in relation to the regrowth. The culm elongation rate was not influenced by $\mathrm{N}$ levels. The

Recebido para publicação em 10/05/2011 e aprovado em 16/04/2013.

'Parte da monografia do primeiro autor. Pesquisa financiada pelo MEC/SESu.

2Engenheiro-Agrônomo, Mestre. Departamento de Zootecnia, Universidade Federal do Ceará, Avenida Mister Hull, s/n, 60970-355, Fortaleza, Ceará, Brasil. nevesvv@ yahoo.com.br ${ }^{3}$ Engenheiro-Agrônomo, Doutor. Departamento de Zootecnia, Universidade Federal do Ceará, Avenida Mister Hull, s/n, 60970-355, Fortaleza, Ceará, Brasil. magno@ufc.br (Pesquisador do CNPQ).

${ }^{4}$ Engenheiro-Agrônomo, Doutor. Empresa Brasileira de Pesquisa Agropecuária, Centro Nacional de Pesquisa Caprinos e Ovinos, Estrada Sobral/Groaíras, Km 4, 62010-970 Sobral, Ceará, Brasil.rpompeu@cnpc.embrapa.br

'Engenheiro-Agrônomo, Doutor. Instituto Federal de Educação, Ciência e Tecnologia, Campus Avançado do Tauá, Avenida Antônio Teixeira Benevides, 01, 63660-000, Tauá, Ceará, Brasil. rodrigogregorio@ hotmail.com

${ }^{6}$ Engenheiros-Agrônomos. Departamento de Engenharia Agrícola, Universidade Federal do Ceará, Avenida Mister Hull, s/n, 60970-355, Fortaleza, Ceará, Brasil. josewellingtonlopes@bol.com.br; ronaldoagroufc@gmail.com

${ }^{7}$ Engenheiro-Agrônomo, Doutor. Departamento de Engenharia Agrícola, Universidade Federal do Ceará, Avenida Mister Hull, s/n, 60970-355, Fortaleza, Ceará, Brasil. cfeitosa@ ufc.br ${ }^{8}$ Engenheiro-Agrônomo, Doutor. Departamento de Engenharia Agrícola, Universidade Federal do Ceará, Avenida Mister Hull, s/n, 60970-355, Fortaleza, Ceará, Brasil. mbezerra@ufc.br 
establishment growth presented higher values than regrowth. The leaf senescence rate before the cut was not influenced by the nitrogen fertilization, however it was altered by the growth cycles. The number of living leaves per tiller was superior on plants fertilized with highest $\mathrm{N}$ levels and at establishment growth, however an opposite effect was

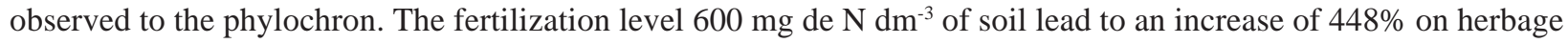
growth, and of $455 \%$ on herbage accumulation rates in relation to the $0 \mathrm{mg} \mathrm{de} \mathrm{N} \mathrm{dm}^{-3}$ of soil. The nitrogen fertilization provides positive responses to the biomass flow of Massai grass.

Key words: morphogenesis, nitrogen, Panicum maximum, phylochron.

\section{INTRODUÇÃO}

A demanda por volumosos que apresentem boa produtividade e qualidade tem aumentado, nos últimos anos, com as restrições cada vez maiores ao desbravamento de novas áreas para a pecuária. Por esse motivo, no ano de 2001, a Embrapa Gado de Corte lançou o cultivar Massai, oriundo do cruzamento entre as espécies Panicum maximum $x$ Panicum infestum, sendo uma gramínea bastante promissora para o pastejo de animais de pequeno porte, tendo merecido atenção pela elevada capacidade de emitir folhas e perfilhos, com rápida rebrotação, após corte ou pastejo.

A produção de biomassa de uma pastagem pode ser melhorada pela utilização de maiores doses de fertilizantes, principalmente os nitrogenados, promovendo expressivo aumento no fluxo de biomassa (Alexandrino et al., 2004; Silva et al., 2009), constituindo prática desejável para o aumento da produção e para a otimização do uso das pastagens (Andrade et al., 2000).

A fase de estabelecimento das forrageiras é de grande relevância para a formação de uma pastagem bem estruturada, caracterizada por perfilhos vigorosos e com capacidade de persistência na comunidade vegetal. O manejo inicial visa a proporcionar uma rebrotação vigorosa nos ciclos posteriores, favorecer a perenidade do pasto, obter elevada produção de matéria seca com boa qualidade, minimizando perdas por senescência e elevando a eficiência de uso da forragem produzida.

Os processos de formação e desenvolvimento de folhas são fundamentais para o crescimento vegetal, e dependem de fatores bióticos e abióticos, como água, luz, temperatura e nutrientes. A taxa de aparecimento de folhas exerce papel central no fluxo de biomassa, em função de sua influência direta sobre os componentes estruturais do pasto (Chapman \& Lemaire, 1993).

O nitrogênio tem papel fundamental para a nutrição das plantas, por ser constituinte essencial de proteínas e pigmentos, tendo grande influência sobre os processos fisiológicos da planta. Esse nutriente participa diretamente de inúmeras etapas da fotossíntese (captação de luz, fixação do dióxido de carbono etc.), bem como de inúmeros outros processos metabólicos da planta (CabreraBosquet et al., 2009).

Por essas razões, este estudo foi conduzido com objetivo de avaliar o efeito da adubação nitrogenada sobre o fluxo de biomassa em Panicum maximum x Panicum infestum cv. Massai, durante o estabelecimento e dois ciclos de rebrotação.

\section{MATERIAL E MÉTODOS}

A pesquisa foi conduzida em casa de vegetação, pertencente ao Departamento de Fitotecnia da Universidade Federal do Ceará - UFC, em Fortaleza - CE, no período de junho a dezembro de 2008. A cidade de Fortaleza/CE está localizada a uma altitude média de 21 metros, com as seguintes coordenadas geográficas: latitude sul de $03^{\circ} 45^{\prime}$ $47^{\prime}$, longitude oeste de $38^{\circ} 31^{\prime} 23$ ", com clima do tipo Aw', tropical chuvoso, segundo classificação de Köeppen. Diariamente, foram registradas as temperaturas mínimas e máximas, apresentando valores médios de 25,8 e $40,5^{\circ} \mathrm{C}$ (estabelecimento), 25,6 e 40,6 ${ }^{\circ} \mathrm{C}$ (rebrotação 1), 25,9 e $40,9^{\circ} \mathrm{C}$ (rebrotação 2), respectivamente.

Utilizou-se o delineamento inteiramente casualizado, em arranjo em parcelas subdivididas, com medidas repetidas no tempo, com cinco repetições. As doses de aduba-

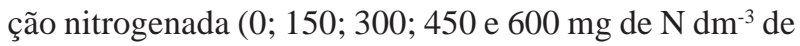
solo) constituíram as parcelas. Os ciclos de crescimento (três ciclos de crescimento: estabelecimento com 43 dias e as rebrotações 1 e 2 com 28 dias cada) foram alocados nas subparcelas.

Os dados da composição química do solo utilizado no experimento encontram-se na Tabela 1. O solo foi corrigido conforme recomendação de CFSEMG (1999), para níveis de fertilidade sugeridos para gramíneas de alto potencial produtivo e com alto nível de produção. Para isto, em vasos com capacidade de $10 \mathrm{dm}^{3}$ cada, aplicou-se uma dose de calcário calcítico de 6,17 g/vaso, equivalente a $1.233 \mathrm{~kg} \mathrm{ha}^{-1}$, de acordo com análise de fertilidade, buscando-se a elevação do teor de cálcio e do $\mathrm{pH}$. Durante um período de dez dias, as amostras 
receberam irrigação diária, visando-se a acelerar a reação do corretivo (Tabela 1).

O controle da lâmina de irrigação diária aplicada foi feito com a instalação de dois tensiômetros de mercúrio $(\mathrm{Hg})$, nos vasos, por tratamento, sendo efetuada a reposição de água, quando a coluna de mercúrio (h) atingia aproximadamente $10 \mathrm{~cm}$, nos tratamentos supridos com maiores doses de nitrogênio. A dose de $600 \mathrm{mg}$ de $\mathrm{N} \mathrm{dm}^{-3}$ de solo foi a referência para a reposição das lâminas aplicadas nas demais doses, segundo a equação: $\psi_{\mathrm{m}}=-12,6 \mathrm{~h}+$ h1 + z; em que: $\psi_{\mathrm{m}}=$ potencial mátrico; $\mathrm{h}=$ altura da coluna de mercúrio na cuba $(\mathrm{cm}) ; \mathrm{h} 1$ = altura do nível de Hg na cuba $(\mathrm{cm})$, em relação à superfície do solo e $\mathrm{z}=$ profundidade da cápsula porosa $(10 \mathrm{~cm})$ (Amaro Filho et al., 2008). Ao final da irrigação, registrou-se a altura da coluna de $\mathrm{Hg}$ na cuba $(\mathrm{cm})$, que se apresentou com aproximadamente $5,0 \mathrm{~cm}$ para todos os tratamentos, ou seja, as condições de umidade no solo eram mantidas próximas à capacidade de campo em todas as unidades experimentais.

A semeadura foi realizada, utilizando-se, em média, 50 sementes por vaso, sendo efetuado o pré-desbaste oito dias após a germinação, permanecendo 12 plantas por vaso, que foram reduzidas para três após o desbaste final, executado 13 dias após a emergência.

Foram realizados três cortes, sendo o primeiro de uniformização, tendo como referência o comprimento horizontal do pseudocolmo, quando todas as plantas dos vasos foram cortadas, deixando um resíduo de $10 \mathrm{~cm}$ de comprimento de colmo, 43 dias após a semeadura. O segundo e o terceiro cortes obedeceram ao mesmo critério e foram realizados após um período de descanso de 28 dias.

As adubações fosfatadas (superfosfato simples), potássica (cloreto de potássio) e micronutrientes (FTE BR-12) foram realizadas de acordo com os resultados da análise do solo, assim como a aplicação do calcário calcítico. As aplicações de nitrogênio (ureia) e de potássio foram parceladas. No crescimento de estabelecimento (43 dias de crescimento), a dose de nitrogênio para cada tratamento foi dividida em duas, sendo a primeira metade aplicada logo após o desbaste final e, a segunda, após 14 dias. Em todas as aplicações de nitrogênio, fez-se a diluição da ureia na água utilizada para a irrigação, buscandose uma melhor uniformização de aplicação do fertilizante.

O potássio foi disponibilizado em duas aplicações, sendo a primeira ( $120 \mathrm{mg} \mathrm{dm}^{-3} \mathrm{de}_{2} \mathrm{O}$ ) realizada logo na implantação (no momento da semeadura). A segunda aplicação de potássio (120 $\mathrm{mg} \mathrm{dm}^{-3}$ de $\mathrm{K}_{2} \mathrm{O}$ ), via solução aquo- sa, foi realizada logo após o corte de uniformização, juntamente com a primeira dose de nitrogênio, na rebrotação 1 .

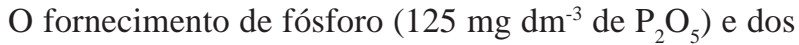
micronutrientes (25 $\mathrm{mg} \mathrm{dm}^{-3}$ de FTE BR-12) foi realizado de uma só vez, no ato da semeadura. A segunda metade da dose de nitrogênio para cada tratamento, aplicada na rebrotação 1 , foi disponibilizada na metade do período de descanso (ciclos de 28 dias). Na rebrotação 2, seguiu-se o mesmo manejo.

Foram identificados três perfilhos, em cada unidade experimental. Cada um dos perfilhos recebeu identificação com um anel de cor diferenciada, para posterior acompanhamento. Os perfilhos marcados foram avaliados a cada três dias, registrando-se o comprimento final das folhas expandidas e emergentes e da porção senescente das expandidas, a largura das folhas, a altura e o comprimento do pseudocolmo e o número de folhas vivas por perfilho. O número de perfilhos por vaso foi quantificado na mesma ocasião das medições anteriores.

As características avaliadas foram: taxa de alongamento foliar (TAlF, cm/perf/dia); taxa de alongamento das hastes (TAlH, cm/perf/dia); filocrono, em número de dias; número de folhas vivas por perfilho (NFV); taxa de senescência foliar anterior (TSFant, cm/perf/dia), contabilizada considerando-se somente as lâminas foliares produzidas antes de cada corte e constituintes da área foliar residual; taxa de senescência foliar posterior (TSFpost, cm/perf/dia), contabilizada considerando-se somente as novas lâminas foliares produzidas durante o período de descanso corrente; taxa de produção de forragem (TPF, g/vaso/dia) e taxa de acúmulo de forragem (TAF, g/vaso/dia). Essas duas últimas variáveis (TPF e TAF) só foram estudadas durante o crescimento de rebrotação 2.

Os dados foram submetidos à análise de variância, teste de comparação de médias e análise de regressão. A interação adubação nitrogenada $\mathrm{x}$ ciclos de crescimento foi desdobrada, quando significativa $(\mathrm{P}<0,05)$, pelo teste F. Os ciclos de crescimento foram comparados pelo teste de Tukey $(\mathrm{P}>0,05)$. O efeito das doses de adubo nitrogenado foi avaliado por análise de regressão, no efeito principal, quando não houve interação, ou no efeito condicionado, quando este ocorreu. A escolha dos modelos baseou-se na significância dos coeficientes linear e quadrático, por meio do teste " $t$ ", de Student $(\mathrm{P}<0,05)$ e no coeficiente de determinação. Como ferramenta de auxílio às análises estatísticas, adotou-se o procedimento GLM, do programa estatístico SAS (SAS Institute, 2003).

Tabela 1. Características químicas do solo ao início da instalação do experimento

\begin{tabular}{|c|c|c|c|c|c|c|c|c|c|c|c|c|c|}
\hline$\overline{\mathbf{P}}$ & $\mathbf{K}$ & $\mathrm{Al}^{3+}$ & $\mathbf{N a}^{+}$ & $\mathrm{Fe}^{2+}$ & $\mathrm{Cu}^{2+}$ & $\mathbf{Z n}^{2+}$ & Mn & $\mathrm{pH}$ & $\mathrm{Ca}^{2+}$ & $\mathbf{M g}^{2+}$ & SB & CTCt & MO \\
\hline \multicolumn{8}{|c|}{$\mathrm{mg} \mathrm{dm}^{-3}$} & $\mathrm{H}_{2} \mathrm{O}$ & \multicolumn{4}{|c|}{$\mathrm{cmol}_{\mathrm{c}} \mathrm{dm}^{-3}$} & $\mathrm{~g} \mathrm{~kg}^{-1}$ \\
\hline 4,0 & 76,0 & 0,0 & 11,0 & 19,0 & 0,14 & 3,91 & 12,18 & 5,7 & 2,0 & 1,9 & 4,14 & 4,14 & 9,1 \\
\hline
\end{tabular}




\section{RESULTADOS E DISCUSSÃO}

Constatou-se interação $(\mathrm{P}<0,05)$, entre doses de nitrogênio $\mathrm{x}$ ciclos de crescimento, para a taxa de alongamento foliar (TAlF), sendo esta taxa incrementada $(\mathrm{P}<0,05)$ nas maiores doses de adubação nitrogenada, e alterada $(\mathrm{P}<0,05)$, entre os ciclos de crescimento (Tabela 2). A TAlF mostrou valor superior $(\mathrm{P}<0,05)$ no estabelecimento, em relação ao seu valor nas rebrotações, apresentando-se semelhante $(\mathrm{P}>0,05)$, em ambas as rebrotações, para as

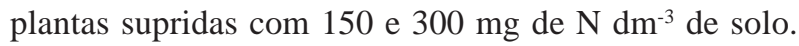
Verificou-se menor valor $(\mathrm{P}<0,05)$ da TAlF na rebrotação 1, para as plantas adubadas com 450 e $600 \mathrm{mg}^{\mathrm{de}} \mathrm{N} \mathrm{dm}^{-3} \mathrm{de}$ solo e em ausência de adubação nitrogenada (Tabela 2). Essa redução da TAlF no referido ciclo de crescimento (rebrotação 1) pode ser atribuída a um elevado impacto do corte sobre a planta, em função de sua estrutura ainda não estar consolidada (Tabela 2) .

A taxa de alongamento foliar respondeu crescentemente $(\mathrm{P}<0,05)$ ao incremento de nitrogênio (Figura 1), nos três ciclos de crescimento (estabelecimento, rebrotação 1 e rebrotação 2). Verificou-se que a TAlF variou de 6,71 a 7,64 cm/perf/dia (estabelecimento) e 0,94 a $2,14 \mathrm{~cm} /$ perf/dia (rebrotação 1) e 1,45 a 2,65 cm/perf/dia (rebrotação 2), para as doses de 0 a $600 \mathrm{mg} \mathrm{dm}^{-3}$ de $\mathrm{N}$, respectivamente, com um incremento de $13,84 \%$ (estabelecimento); $127,7 \%$ e (rebrotação 1) e 82,8\% (rebrotação 2) para a dose de $600 \mathrm{mg} \mathrm{dm}^{-3} \mathrm{de} \mathrm{N}$, em relação a seus valores na ausência de adubação nitrogenada (Figura 1), demonstrando o significativo papel desse nutriente no comportamento dessa variável, podendo ser atribuído à deposição de nutrientes, especialmente de nitrogênio, nas zonas de alongamento e divisão celular das folhas (Skinner \& Nelson, 1995) (Figura 1) .

Dentre os efeitos benéficos da adubação nitrogenada, destaca-se o estímulo ao desenvolvimento dos primórdios foliares, o aumento do número de folhas vivas por perfilho (Silveira \& Monteiro, 2007), a diminuição do intervalo de tempo para aparecimento de folhas (Patês et al., 2007; Garcez Neto et al., 2002) e o aumento do perfilhamento (Lavres Júnior \& Monteiro, 2003).

A taxa de alongamento foliar é uma variável de grande relevância na análise do fluxo de biomassa das plantas, visto que, à medida que a TAlF aumenta, ocorre incremento na proporção de folhas e, consequentemente, maior área foliar fotossinteticamente ativa, promovendo maior acúmulo de biomassa.

Não foi constatada interação $(\mathrm{P}>0,05)$, entre os fatores doses de nitrogênio $\mathrm{x}$ ciclos de crescimento, para a taxa de alongamento das hastes (TAlH), tampouco, verificou-se efeito $(\mathrm{P}>0,05)$ das doses de nitrogênio sobre esta variável, com média de $0,166 \mathrm{~cm} /$ perf/dia (Tabela 2). A ausência de incremento na taxa de alongamento das hastes, nas doses elevadas de nitrogênio, demonstra a genética positiva do capim-massai, em sistema intensivo de produção, pelo fato de a forrageira responder preferencialmente em produção de biomassa foliar. A TAlH diferiu $(\mathrm{P}<0,05)$ entre os períodos de crescimento, apresentando maior valor $(0,380 \mathrm{~cm} /$ perf/dia) durante o crescimento em estabelecimento, por causa do prolongado período de descanso, de 43 dias (Tabela 2).

Tabela 2. Características morfogênicas em Panicum maximum x Panicum infestum cv. Massai durante três ciclos de crescimento (estabelecimento, rebrotação 1 e rebrotação 2) adubado com nitrogênio

\begin{tabular}{|c|c|c|c|c|c|c|}
\hline \multirow{2}{*}{ Ciclos } & \multicolumn{6}{|c|}{ Doses de nitrogênio $\left(\mathrm{mg} \mathrm{dm}^{-3}\right)$} \\
\hline & $\mathbf{0}$ & 150 & 300 & 450 & 600 & Média \\
\hline \multicolumn{7}{|c|}{ Taxa de alongamento foliar (TAlF, cm/perf/dia) } \\
\hline Estabelecimento & $6,65^{\mathrm{Ad}}$ & 6,93 Acd & $7,17 \mathrm{Acb}$ & $7,73^{\mathrm{Aa}}$ & $7,41^{\text {Bab }}$ & 7,18 \\
\hline Rebrotação 1 & $0,84^{\mathrm{Cc}}$ & $1,31 \mathrm{Bb}$ & 1,68 Bab & $2,00 \mathrm{Ca}$ & $2,09 \mathrm{Ca}$ & 1,58 \\
\hline Rebrotação 2 & $1,38^{\mathrm{Bd}}$ & $1,65^{\mathrm{Bcd}}$ & $1,97 \mathrm{Bbc}$ & $2,46^{\mathrm{Ba}}$ & $2,20^{\mathrm{Bab}}$ & 1,93 \\
\hline \multicolumn{7}{|c|}{ Taxa de alongamento das hastes (TAlH, cm/perf/dia) } \\
\hline Estabelecimento & 0,351 & 0,364 & 0,373 & 0,405 & 0,408 & $0,380^{\mathrm{A}}$ \\
\hline Rebrotação 1 & 0,034 & 0,050 & 0,044 & 0,053 & 0,054 & $0,047^{\mathrm{C}}$ \\
\hline Rebrotação 2 & 0,045 & 0,048 & 0,071 & 0,098 & 0,093 & $0,071^{\text {в }}$ \\
\hline \multicolumn{7}{|c|}{ Taxa de senescência foliar anterior (TSFant, $\mathrm{cm} /$ perf/dia) } \\
\hline Rebrotação 1 & 0,004 & 0,004 & 0,002 & 0,00 & 0,00 & $0,0012^{\mathrm{B}}$ \\
\hline Rebrotação 2 & 0,012 & 0,008 & 0,020 & 0,020 & 0,012 & $0,0144^{\mathrm{A}}$ \\
\hline \multicolumn{7}{|c|}{ Taxa de senescência foliar posterior (TSFpost, $\mathrm{cm} /$ perf/dia) } \\
\hline Estabelecimento & 2,732 & 2,640 & 2,592 & 2,628 & 2,738 & $2,666^{\mathrm{A}}$ \\
\hline Rebrotação 1 & 0,000 & 0,004 & 0,008 & 0,004 & 0,010 & $0,005^{\text {в }}$ \\
\hline Rebrotação 2 & 0,040 & 0,048 & 0,058 & 0,080 & 0,062 & $0,058^{\text {в }}$ \\
\hline
\end{tabular}

Médias seguidas de letras iguais, na mesma linha (minúsculas) e na mesma coluna (maiúsculas), não diferem (P>0,05), pelo teste de Tukey.

Rev. Ceres, Viçosa, v. 60, n.3, p. 363-371, mai/jun, 2013 
A taxa de alongamento das hastes é uma variável morfogênica de grande relevância para o crescimento, pois garante a manutenção da arquitetura do dossel, quando este atinge uma biomassa mais elevada, mantendo o distanciamento adequado entre as folhas e evitando um aumento no coeficiente de extinção luminosa (Sugiyama et al., 1985). Por outro lado, apresenta efeitos negativos na qualidade da forragem produzida (Cândido et al., 2006; Silva et al., 2007a) e no seu aproveitamento pelos animais em pastejo (Silva et al., 2007b).

Não houve interação, $(\mathrm{P}>0,05)$, entre doses de nitrogênio $\mathrm{x}$ ciclos de crescimento, para a taxa de senescência foliar anterior (TSFant). A TSFant não foi influenciada $(\mathrm{P}<0,05)$ pela adubação nitrogenada, com média de 0,078 $\mathrm{cm} /$ perf/dia; porém, verificou-se diferença $(\mathrm{P}<0,05)$ entre ambas as rebrotações (Tabela 2). O crescimento em rebrotação 2 apresentou $0,0144 \mathrm{~cm} /$ perf/dia de senescência, mostrando-se, seu valor, superior ao valor observado na rebrotação $1(0,0012 \mathrm{~cm} /$ perf/dia $)$.

A taxa de senescência foliar anterior pode ser um indicativo, em grande parte, da intensidade de corte ou pastejo adotada, na medida em que um pastejo mais intenso reflete-se em menor área foliar residual e, consequentemente, em menor quantidade de folhas que entrarão em senescência, reduzindo a TSFant. Também pode ser um indicativo, embora em menor proporção, de frequência de pastejo, uma vez que períodos de descanso prolongados demais poderão ocasionar a senescência das primeiras folhas produzidas na rebrotação. Essas folhas, quando já mortas, serão rejeitadas pelo animal em pastejo e participarão da quantificação da senescência das folhas produzidas no período de descanso anterior (Pompeu et al., 2009).
Não foi constatada interação $(\mathrm{P}>0,05)$, entre doses de nitrogênio $\mathrm{x}$ ciclos de crescimento, para a taxa de senescência foliar posterior (TSFpost) (Tabela 2). A TSFpost não foi influenciada $(\mathrm{P}>0,05)$ pelas doses de nitrogênio, com média de $0,910 \mathrm{~cm} /$ perf/dia. Constatou-se diferença $(\mathrm{P}<0,05)$ para esta variável entre os ciclos de crescimento, com o estabelecimento apresentando TSFpost de 2,67 cm/perf/dia, mostrando-se seu valor superior ao das rebrotações 1 e 2 (0,005 e $0,058 \mathrm{~cm} /$ perf/dia, respectivamente). A idade de crescimento mais avançada durante o estabelecimento (43 dias) justifica esta observação, por permitir maior tempo de acompanhamento da senescência.

O próprio ritmo de crescimento da planta, respondendo às doses crescentes de nitrogênio, com altas taxas de aparecimento e alongamento foliares, favorece a senescência das folhas primeiramente formadas, uma vez que aumenta a competição por fotoassimilados (Gomide et al., 2003). Maiores taxas de senescência foliar $(5,5 \mathrm{~cm} /$ perf/dia) durante o crescimento de estabelecimento foram constatadas por Gomide et al. (2003), em estudos conduzidos com o capim-mombaça, durante o estabelecimento e a rebrotação. Essa comparação demonstra a genética positiva do material estudado, por persistir com baixa taxa senescência foliar posterior ao corte, com a intensificação no uso de fertilizantes nitrogenados, visto que o nitrogênio acelera vários processos fisiológicos nas plantas.

A taxa de senescência foliar posterior é um indicativo do ajuste da frequência de desfolhação à fisiologia do dossel, pois um pasto manejado para alta eficiência de uso da forragem produzida deve prevenir a senescência de folhas formadas na rebrotação, ou seja, deve apresentar uma TSFpost igual ou próxima de zero (Cândido et al., 2006).

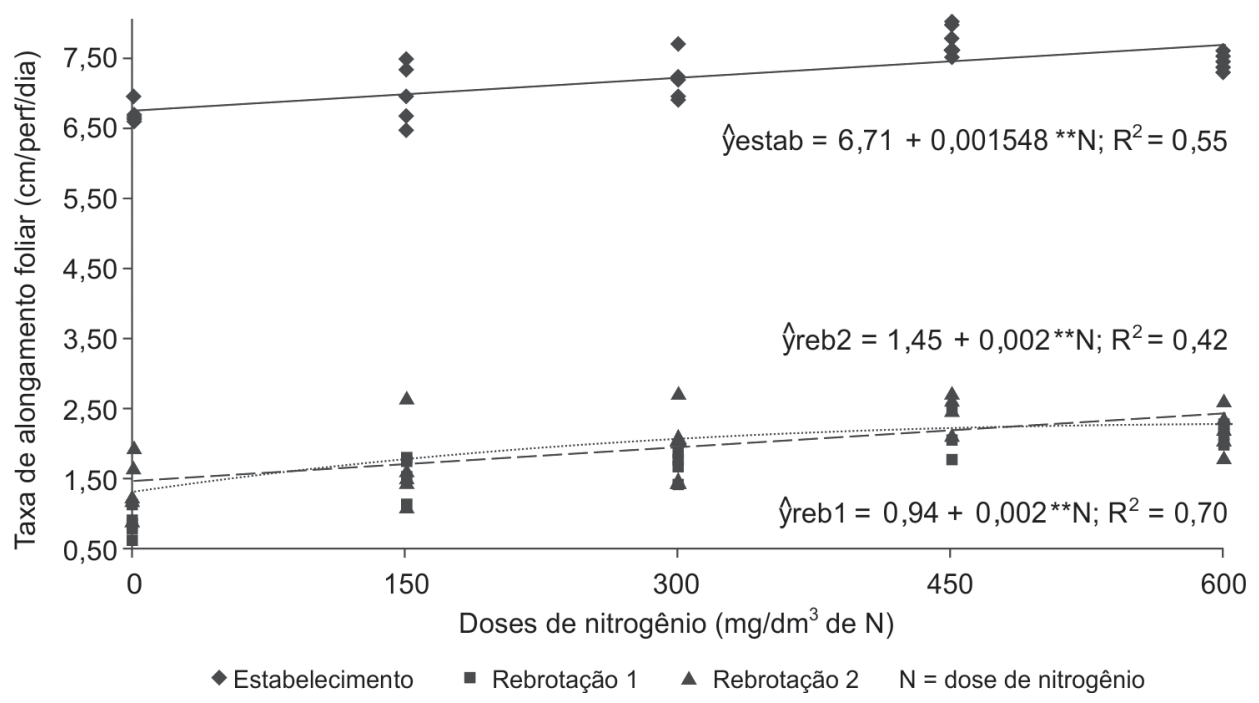

Figura 1. Efeito das doses de nitrogênio sobre a taxa de alongamento foliar ( $\mathrm{cm} /$ perf/dia) durante os três ciclos de crescimento (estabelecimento, rebrotação 1 e rebrotação 2); $\hat{y}$ = valores estimados a partir da equação de regressão para cada variável analisada; significativo a $1 \%(* *)$. 
Para a variável número de folhas vivas por perfilho (NFV), constatou-se interação $(\mathrm{P}<0,05)$ entre os fatores (doses de nitrogênio $\mathrm{x}$ ciclos de crescimento), sendo esta variável aumentada $(\mathrm{P}<0,05)$ nas maiores doses de nitrogênio e diminuída $(\mathrm{P}<0,05)$ do estabelecimento para as rebrotações, tendendo a estabilizar-se nas rebrotações 1 e 2 (Tabela 3).

O número de folhas vivas por perfilho respondeu linearmente $(\mathrm{P}<0,05)$, com o aumento das doses de nitrogênio, no estabelecimento e na rebrotação 1, e apresentou resposta quadrática $(\mathrm{P}<0,05)$ na rebrotação 2 (Figura 2).

Observou-se que o NFV por perfilho variou de 3,50 a 4,00 (estabelecimento), 1,42 a 2,62 (rebrotação 1) e 1,41 a 2,49 (rebrotação 2), para as doses de 0 a $600 \mathrm{mg} \mathrm{dm}^{-3} \mathrm{de} \mathrm{N}$, respectivamente, com um incremento de $14,3 \%$ (estabelecimento), 84,5\% (rebrotação 1) e 76,6\% (rebrotação 2), para a dose de $600 \mathrm{mg} \mathrm{dm}^{-3}$ de $\mathrm{N}$, em relação a seus valores na ausência de adubação nitrogenada. Para cada $1 \mathrm{mg}$ de $\mathrm{N}$ $\mathrm{dm}^{-3}$ de solo, observou-se incrementos de 0,00083; 0,002 e 0,02998 folhas por perfilho, para estabelecimento, rebrotação 1 e 2, respectivamente (Figura 2).

Nesse contexto, o número total de folhas vivas por perfilho expressa o potencial de assimilação de carbono e de produção de forragem ao nível de perfilho. Essa variável apresenta, ainda, grande importância na avaliação e manejo das plantas forrageiras, por tratar-se do componente da biomassa com melhor atributo qualitativo, sendo a fração mais selecionada pelos animais em pastejo e por sua aplicação, como critério de definição prático para a determinação do período de descanso, numa área de pastejo (Fulkerson \& Donaghy, 2001). Com isso, a elevação da adubação nitrogenada pode antecipar o momento de desfolhação da planta. A definição do momento certo de desfolhação deve basear-se no objetivo do manejo adotado na pastagem, evidenciando a fisiologia da forrageira; porém, a adubação nitrogenada vai interferir nesse momento, pois influencia na morfofisiologia da planta.
O filocrono foi influenciado, tanto pela adubação nitrogenada, quanto pelos ciclos de crescimento, com interação $(\mathrm{P}<0,05)$ entre esses fatores. Essa variável decresceu $(\mathrm{P}<0,05)$ nas maiores doses e apresentou valor inferior, no estabelecimento, em relação aos valores nas rebrotações (Tabela 3). Durante o estabelecimento, verificou-se resposta linear decrescente $(\mathrm{P}<0,05)$, com o incremento nas doses de nitrogênio, com valores estimados em 7,74 e 6,83 dias para 0 e $600 \mathrm{mg} \mathrm{dm}^{-3}$ de $\mathrm{N}$, respectivamente. Observou-se resposta quadrática, para as doses de nitrogênio estudadas, em ambas as rebrotações, estimados em 15,92 e 11,12 dias (rebrotação 1) e 15,10 e 10,30

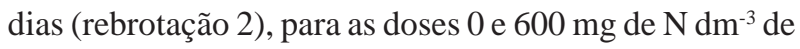
solo, respectivamente, alcançando ponto de mínimo (10,92 e 10,10 dias, rebrotações 1 e 2 , respectivamente), na dose de $500 \mathrm{mg} \mathrm{dm}^{-3}$ de N, para ambas rebrotações (Figura 3).

Esse comportamento, verificado para o filocrono, pode ser atribuído à combinação entre alongamento foliar em proporção superior ao alongamento da bainha, associado à disponibilidade adequada de temperatura e nutrientes.

O menor valor (7,3 dias/folha) para o filocrono foi constatado no crescimento de estabelecimento (Tabela 3), mostrando maior capacidade responsiva dessa planta durante o primeiro ciclo de crescimento. A redução do filocrono com a adubação nitrogenada é decorrente do efeito do nitrogênio sobre as taxas de crescimento, especialmente a foliar (Garcez Neto et al., 2002), conferindo às pastagens maior capacidade de reposição dos tecidos, visto que, após a desfolhação, uma rápida recuperação de seu aparato fotossintético possibilita maior capacidade competitiva dos indivíduos na comunidade vegetal (Martuscello et al., 2006). O nitrogênio assume papel de extrema importância, ao favorecer essa recuperação, pois é um nutriente essencial em vários processos fisiológicos.

As taxas de aparecimento foliar, alongamento foliar e tempo de vida da folha são características determinadas geneticamente, mas podem ser influenciadas pela tempe-

Tabela 3. Características do fluxo de biomassa em Panicum maximum x Panicum infestum cv. Massai submetido a doses crescentes de nitrogênio

\begin{tabular}{|c|c|c|c|c|c|c|}
\hline \multirow{2}{*}{ Ciclos } & \multicolumn{6}{|c|}{ Doses de nitrogênio $\left(\mathrm{mg} \mathrm{dm}^{-3}\right)$} \\
\hline & $\mathbf{0}$ & 150 & 300 & 450 & 600 & Média \\
\hline \multicolumn{7}{|c|}{ Número de folhas vivas por perfilho (NFV, folhas/perfilho) } \\
\hline Estabelecimento & $3,6^{\mathrm{Ad}}$ & $3,5^{\mathrm{Ad}}$ & 3,7 Acd & $4,1^{\mathrm{Aa}}$ & $3,9^{\mathrm{Abc}}$ & 3,8 \\
\hline Rebrotação 1 & $1,4^{\mathrm{Bd}}$ & $1,7^{\mathrm{Bc}}$ & $2,0^{\mathrm{Bb}}$ & $2,1^{\mathrm{Cab}}$ & $2,3^{\mathrm{Ba}}$ & 1,9 \\
\hline \multirow[t]{2}{*}{ Rebrotação 2} & $1,5^{\mathrm{Bc}}$ & $1,7^{\mathrm{Bb}}$ & $2,0^{\mathrm{Bb}}$ & $2,5^{\mathrm{Ba}}$ & $2,3^{\mathrm{Ba}}$ & 2,0 \\
\hline & \multicolumn{6}{|c|}{ Filocrono (dias/folha) } \\
\hline Estabelecimento & $7,6^{\mathrm{Ba}}$ & $7,8 \mathrm{Ba}$ & $7,4^{\text {ва }}$ & $6,6^{\mathrm{Ca}}$ & $7,1^{\mathrm{Ba}}$ & 7,3 \\
\hline Rebrotação 1 & $15,9^{\mathrm{Aa}}$ & $13,3^{\mathrm{Ab}}$ & $10,8^{\mathrm{Ac}}$ & $9,9^{\mathrm{Ac}}$ & $9,4^{\mathrm{Ac}}$ & 11,9 \\
\hline Rebrotação 2 & 14,9 Аа & $12,5^{\mathrm{Ab}}$ & $10,8^{\mathrm{Ac}}$ & $8,4^{\mathrm{Bd}}$ & 9,4 Acd & 11,2 \\
\hline
\end{tabular}

Médias seguidas de letras iguais, na mesma linha (minúsculas) e na mesma coluna (maiúsculas), não diferem (P>0,05), pelo teste de Tukey

Rev. Ceres, Viçosa, v. 60, n.3, p. 363-371, mai/jun, 2013 
ratura e disponibilidade de nitrogênio e de água (Lemaire \& Chapman, 1996). Neste estudo, a elevação das doses de nitrogênio proporcionou incrementos na TAlF e, consequentemente, na TApF, resultando em diminuição no filocrono e, dessa forma, ratificando o efeito desse nutriente sobre essas características (Martuscello et al., 2005; Mesquita \& Neres, 2008; Silva et al., 2009).

As variáveis taxas de produção de forragem (TPF) e de acúmulo de forragem (TAF), estudadas durante o crescimento de rebrotação 2, apresentaram valores superiores $(\mathrm{P}<0,05)$, com a elevação da adubação nitrogenada, revelando um padrão linear crescente, na resposta de ambas as variáveis (Figuras 4 e 5).
Verificou-se que a TPF variou de 0,2084 a 1,142 g/vaso/ dia (Figura 4) e a TAF de 0,1956 a 1,085 g/vaso/dia (Figura 5), para as doses de 0 a $600 \mathrm{mg} \mathrm{dm}^{-3} \mathrm{de} \mathrm{N}$, respectivamente. A dose de $600 \mathrm{mg} \mathrm{dm}^{-3} \mathrm{de} \mathrm{N}$ proporcionou um incremento de $448 \%$ na TPF e $455 \%$ na TAF (rebrotação 2), em relação aos valores do tratamento com ausência de nitrogênio. Para cada $1 \mathrm{mg} / \mathrm{dm}^{3}$ de N, observaram-se incrementos de 0,001556 e 0,001483 g/vaso/dia, para TPF e TAF, respectivamente (Figuras 4 e 5 , respectivamente).

O comportamento mostrado pela TPF ratifica a importância do nitrogênio para o incremento de biomassa, em forrageiras manejadas intensivamente. $\mathrm{O}$ incremento observado na TAlF, com a elevação nas doses de nitrogê-

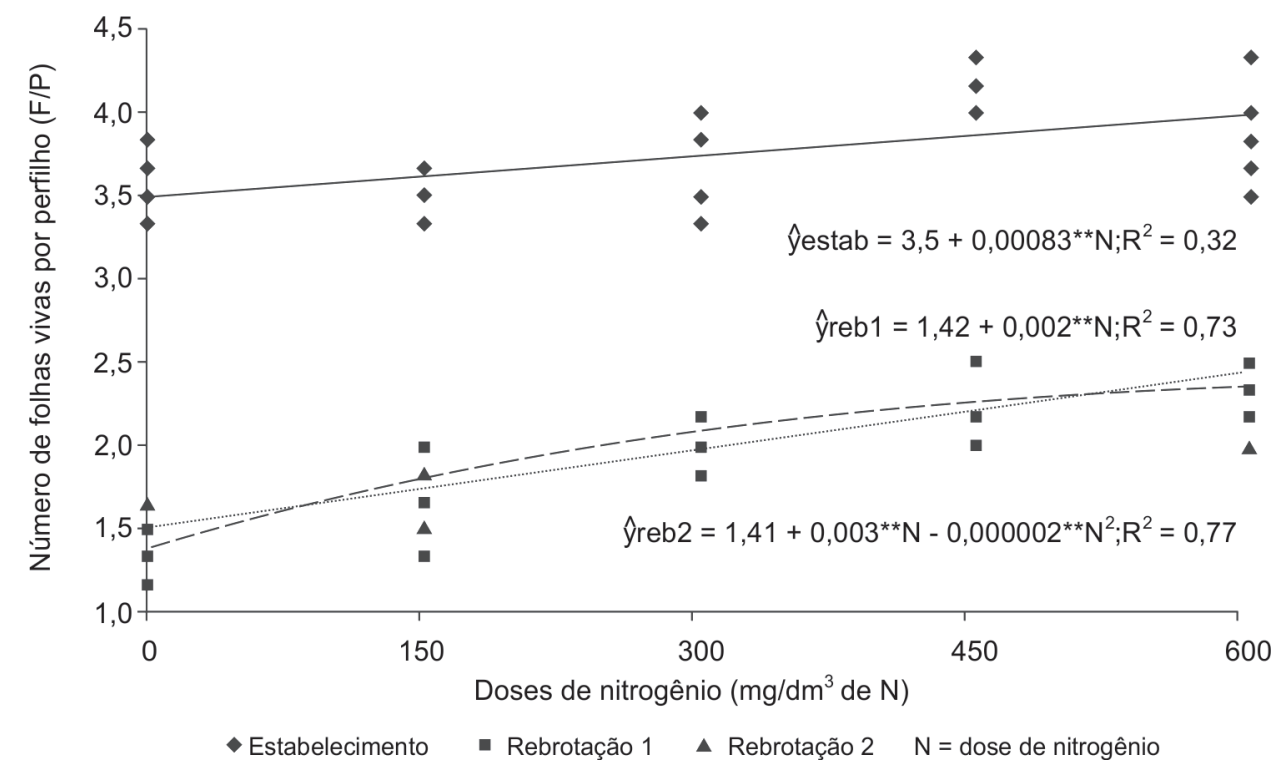

Figura 2. Efeito das doses de nitrogênio sobre o número de folhas vivas por perfilho (folhas/perfilho) durante os três ciclos de crescimento (estabelecimento, rebrotação 1 e rebrotação 2); $\hat{y}$ = valores estimados a partir da equação de regressão para cada variável analisada; significativo a $1 \%(* *)$.

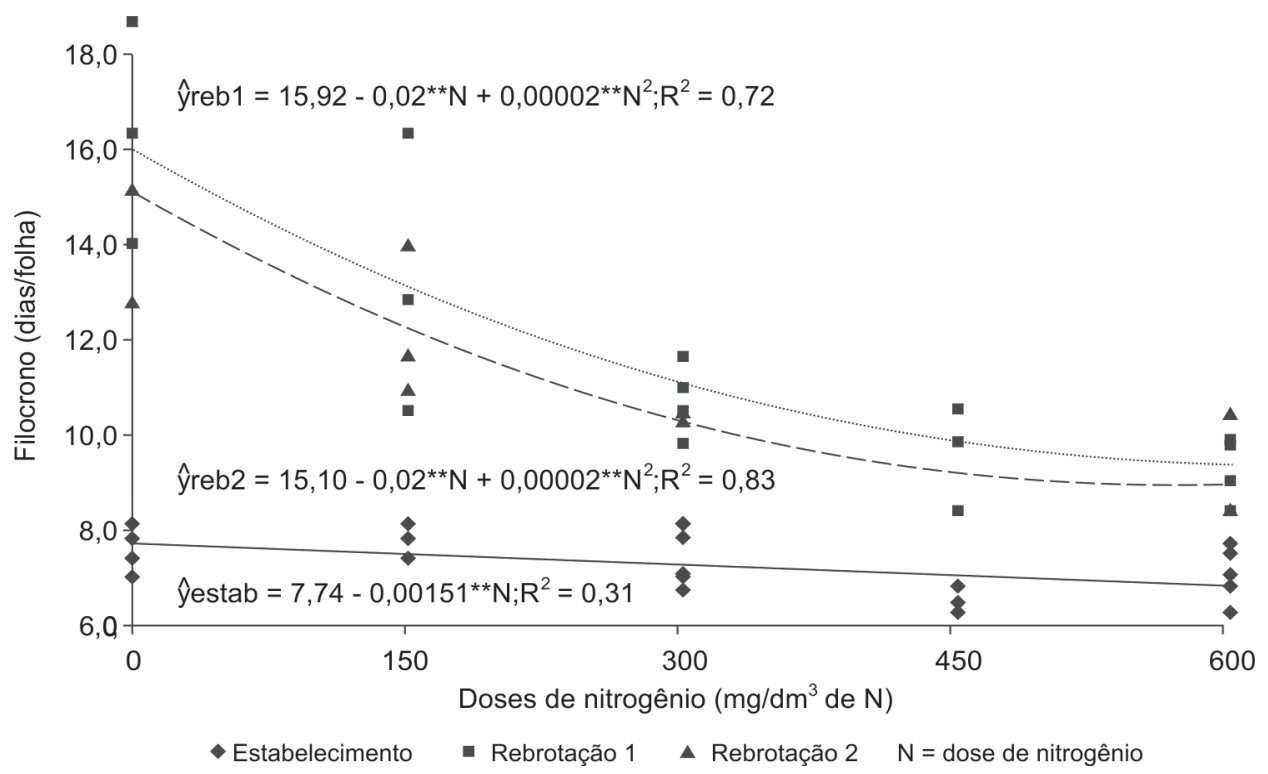

Figura 3. Efeito das doses de nitrogênio sobre o filocrono (dias/folha) durante os três ciclos de crescimento (estabelecimento, rebrotação 1 e rebrotação 2); $\hat{y}$ = valores estimados a partir da equação de regressão para cada variável analisada; significativo a $1 \%$ (**). 


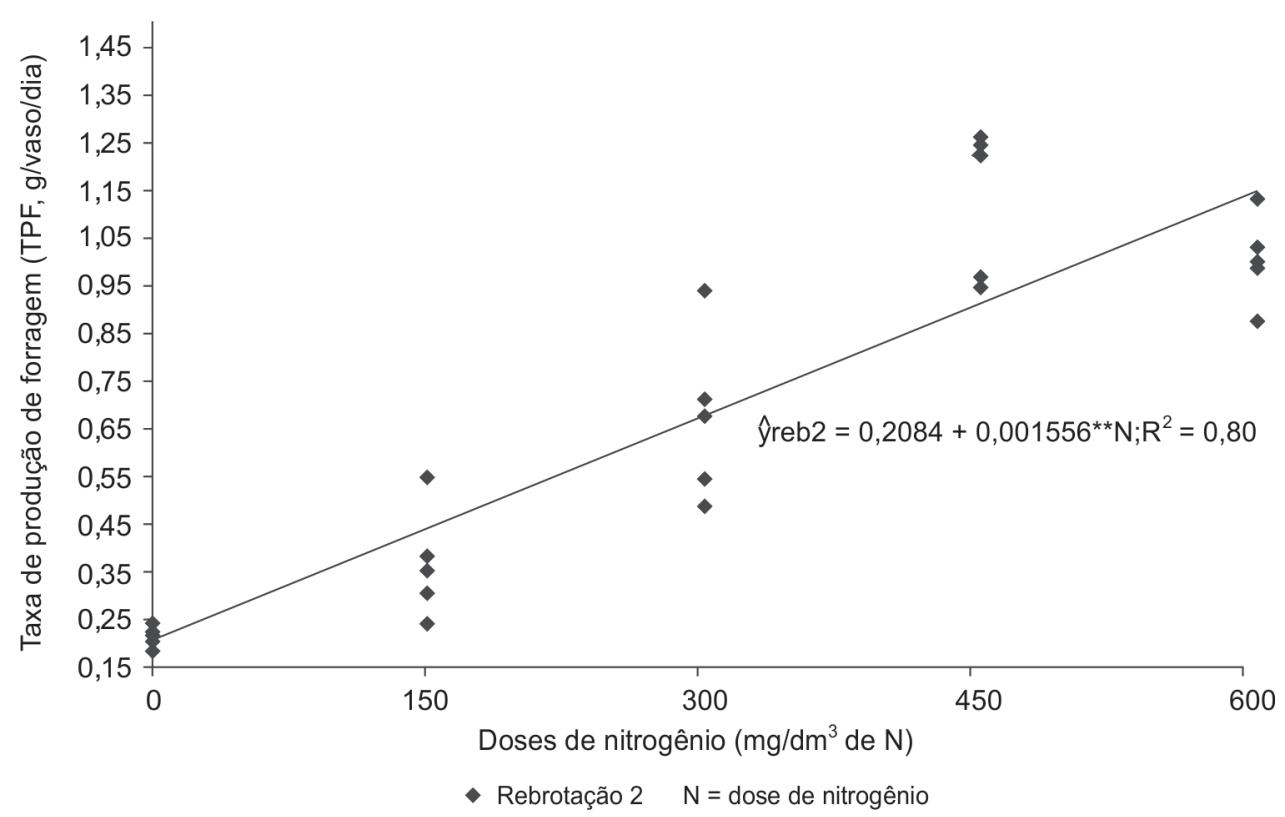

Figura 4. Efeito das doses de nitrogênio sobre a taxa de produção de forragem (g/vaso/dia) durante o crescimento de rebrotação 2; $\hat{y}=$ valores estimados a partir da equação de regressão para a variável analisada; significativo a $1 \%$ (**).

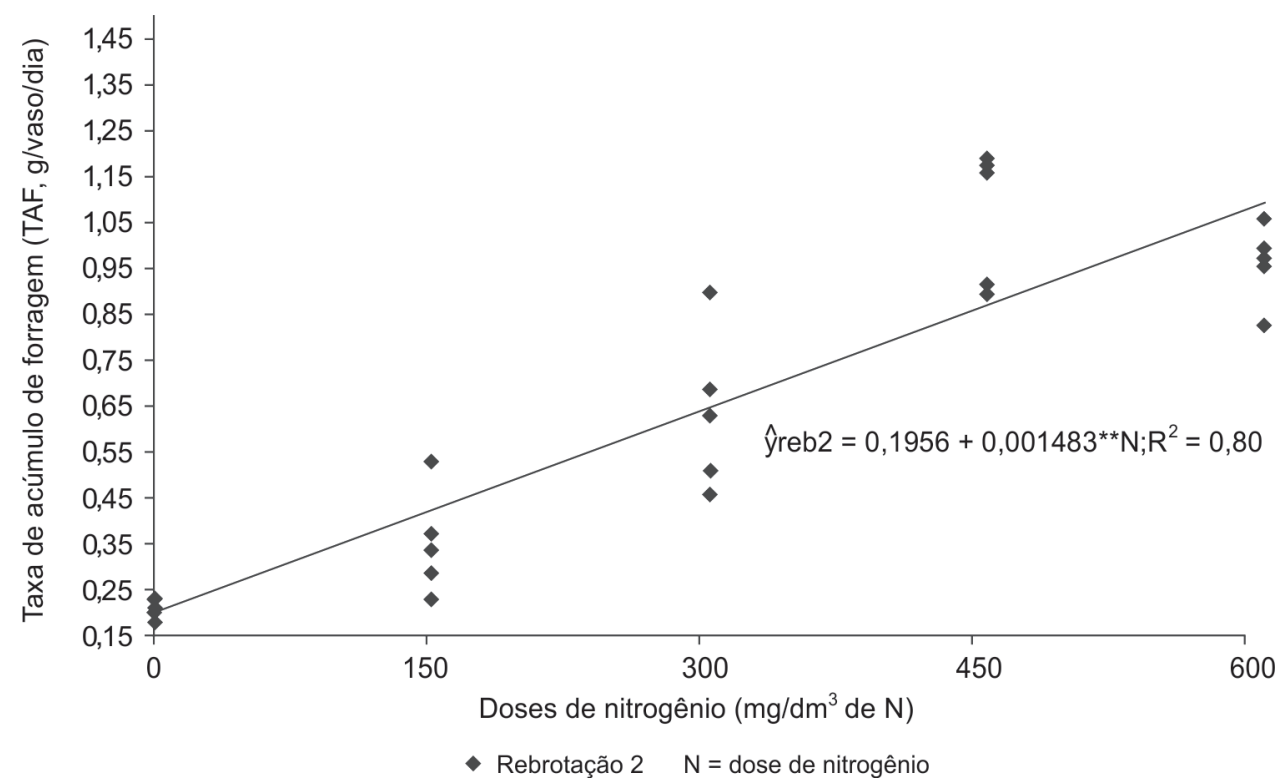

Figura 5. Efeito das doses de nitrogênio sobre a taxa de acúmulo de forragem (g/vaso/dia) durante o crescimento de rebrotação 2; $\hat{y}=$ valores estimados a partir da equação de regressão para a variável analisada; significativo a $1 \%$ (**).

nio, justifica os valores da TPF, visto que ambas têm correlação linear positiva $\left(0,83^{* *}\right)$. O acúmulo de forragem é o resultado do balanço entre os componentes do fluxo de biomassa, individualmente e em nível de comunidade. A pequena diferença entre a TPF e a TAF reflete a baixa senescência do capim-massai, o que pode ser comprovado pela TSFpost, que foi desprezível na rebrotação 2 , com média de $0,058 \mathrm{~cm} /$ perf $/$ dia.

\section{CONCLUSÕES}

A adubação nitrogenada proporciona respostas positivas para o fluxo de biomassa do capim-massai, com desta- que para as variáveis taxa de alongamento foliar, filocrono, número de folhas vivas por perfilho, taxa de produção de forragem e taxa de acúmulo de forragem, podendo-se utilizar uma dose de nitrogênio de até $600 \mathrm{mg} \mathrm{dm}^{-3}$.

As características morfogênicas do capim-massai são modificadas pelos ciclos de crescimento.

\section{AGRADECIMENTOS}

À SESu, especialmente ao Programa de Educação Tutorial (PET), pelo auxílio financeiro e concessão das bolsas. 


\section{REFERÊNCIAS}

Alexandrino E, Nascimento Júnior D, Mosquim PR, Regazzi AJ \& Rocha FC (2004) Características morfogênicas e estruturais na rebrotação da Brachiaria brizantha $\mathrm{cv}$. Marandu submetida a três doses de nitrogênio. Revista Brasileira de Zootecnia, 33:1372-1379.

Amaro Filho J, Assis Júnior RN \& Mota JCA (2008) Física do solo: conceitos e aplicações. $1^{\text {a }}$ ed. Fortaleza, Imprensa Universitária. $290 \mathrm{p}$

Andrade AC, Fonseca DM, Gomide JA, Alvarez VH, Martins CE \& Souza DPH (2000) Produtividade e valor nutritivo do capimelefante cv. napier sob doses crescentes de nitrogênio e potássio. Revista Brasileira de Zootecnia, 29:1589-1595.

Cabrera-Bosquet L, Albrizio R, Araus JL \& Nogués S (2009) Photosynthetic capacity of field-grown durum wheat under different $\mathrm{N}$ availabilities: A comparative study from leaf to canopy. Environmental and Experimental Botany, 67:145-152, 2009 .

Cândido MJD, Silva RG, Neiva JNM, Facó O, Benevides YI \& Farias S.F (2006) Fluxo de biomassa em capim-tanzânia pastejado por ovinos sob três períodos de descanso. Revista Brasileira de Zootecnia, 35:2234-2242

Chapman DF \& Lemaire G (1993) Morphogenetic and structural determinants of plant regrowth after defoliation. In: Baker MJ (Ed.) Grasslands for our world. Wellington, SIR Publishing. p.5564 .

CFSEMG - Comissão de Fertilidade do Solo do Estado de Minas Gerais (1999) Recomendações para uso de corretivos e fertilizantes em Minas Gerais, 5 ${ }^{\text {a }}$ Aproximação. Viçosa, UFV. 359p.

Fulkerson WJ \& Donaghy DJ (2001) Plant soluble carbohydrate reserves and senescence - key criteria for developing an effective grazing management system for ryegrass based pasture: a review. Australian Journal Experimental Agriculture, 41:261275

Garcez Neto AF, Nascimento Júnior D, Regazzi AJ, Fonseca DM, Mosquim PR \& Gobbi KF (2002) Respostas morfogênicas e estruturais de Panicum Maximum cv. Mombaça sob diferentes níveis de adubação nitrogenada e alturas de corte. Revista Brasileira de Zootecnia, 31:1890-1900.

Gomide CAM, Gomide J A \& Alexandrino E (2003) Índices Morfogênicos e de Crescimento durante o Estabelecimento e a Rebrotação do Capim-Mombaça (Panicum maximum Jacq.). Revista Brasileira de Zootecnia, 32:795-803.

Lavres Júnior J \& Monteiro FA (2003) Perfilhamento, área foliar e sistema radicular do capim-Mombaça submetido a combinações de doses de nitrogênio e potássio. Revista Brasileira de Zootecnia, 32:1068-1075.

Lemaire G \& Chapman D (1996) Tissue flows in grazed plant communities. In: Hodgson J \& Illius AW (Eds.) The ecology and management of grazing systems. Wallingford, CAB International. p.3-36.

Martuscello JA, Fonseca DM, Nascimento Júnior D, Santos PM, Junior JIR, Cunha DNFV \& Moreira LM (2005) Características morfogênicas e estruturais do capim-xaraés submetido à adubação nitrogenada e desfolhação. Revista Brasileira de Zootecnia, 34:1475-1482.

Martuscello JÁ, Fonseca DM, Nascimento Júnior D, Santos PM, Cunha DNFV \& Moreira LM (2006) Características morfogênicas e estruturais de capim-massai submetido a adubação nitrogenada e desfolhação. Revista Brasileira de Zootecnia, $35: 665-671$.
Mesquita EE \& Neres MA (2008) Morfogênese e composição bromatológica de cultivares de Panicum maximum em função da adubação nitrogenada. Revista Brasileira de Saúde e Produção Animal, 9:201-209.

Patês NMS, Pires AJV, Silva CCF, Santos LC, Carvalho GGP \& Freire MAL (2007) Características morfogênicas e estruturais do capim-tanzânia submetido a doses de fósforo e nitrogênio. Revista Brasileira de Zootecnia, 36:1736-1741.

Pompeu RCFF, Cândido MJD, Neiva JNM, Rogério MCP, Benevides YI \& Oliveira BCM (2009) Fluxo de biomassa em capim-tanzânia sob lotação rotativa com quatro níveis de suplementação concentrada. Revista Brasileira de Zootecnia, 38:809-817.

SAS Institute. SAS System for Windows. Version 9.0 (2003) Cary: SAS Institute Inc. 2 CD-ROMs.

Silva CCF, Bonomo P, Pires AJV, Maranhão CMA, Patês NMS \& Santos LC (2009) Características morfogênicas e estruturais de duas espécies de braquiária adubadas com diferentes doses de nitrogênio. Revista Brasileira de Zootecnia, 38:657-661.

Silva MJD, Cândido MJD, Neiva JNM, Lôbo RNB \& Silva DS (2007a) Características estruturais do dossel de pastagens de capim-tanzânia mantidas sob três períodos de descanso com ovinos. Revista Brasileira de Zootecnia, 36:1255-1265.

Silva RG, Neiva JNM, Cândido MJD \& Lobo RNB (2007b) Aspectos comportamentais e desempenho produtivo de ovinos mantidos em pastagens de capim-tanzânia manejado sob lotação intermitente. Ciência Animal Brasileira, 8:609-620.

Silveira CP \& Monteiro FA (2007) Morfogênese e produção de biomassa do capim-tanzânia adubado com nitrogênio e cálcio. Revista Brasileira de Zootecnia, 36:335-342.

Skinner RH \& Nelson CJ (1995) Elongation of the grass leaf and its relationship to the phyllochron. Crop Science, 35:4-10.

Sugiyama S, Yoneyama M, Takahashi N \& Gotoh K (1985) Canopy structure and productivity of Festuca arundinaceae Schreb, swards during vegetative and reproductive growth. Grass and Forage Science, 40:49-55. 